\title{
Crystallography and mutagenesis point to an essential role for the N-terminus of human mitochondrial ClpP
}

\author{
Sung Gyun Kang ${ }^{\mathrm{a}}$, Michael R. Maurizi ${ }^{\mathrm{a}, *}$, Mark Thompson ${ }^{\mathrm{b}}$, Timothy Mueser ${ }^{\mathrm{b}}$, \\ Bijan Ahvazi ${ }^{\mathrm{b}, \mathrm{c}, *}$ \\ a Laboratory of Cell Biology, National Cancer Institute, Bethesda, MD 20892-4255, USA \\ ${ }^{\mathrm{b}}$ Laboratory of Structural Biology Research, Bethesda, MD 20892-8023, USA \\ ${ }^{\mathrm{c}}$ X-ray Crystallography Facility, National Institute of Arthritis and Musculoskeletal and Skin Diseases, National Institutes of Health, \\ Bethesda, MD 20892-8023, USA
}

Received 28 May 2004, and in revised form 20 July 2004

Available online 11 September 2004

\begin{abstract}
We have determined a $2.1 \AA$ crystal structure for human mitochondrial ClpP (hClpP), the proteolytic component of the ATPdependent ClpXP protease. HClpP has a structure similar to that of the bacterial enzyme, with the proteolytic active sites sequestered within an aqueous chamber formed by face-to-face assembly of the two heptameric rings. The hydrophobic N-terminal peptides of the subunits are bound within the narrow $(12 \AA)$ axial channel, positioned to interact with unfolded substrates translocated there by the associated ClpX chaperone. Mutation or deletion of these residues causes a drastic decrease in ClpX-mediated protein and peptide degradation. Residues 8-16 form a mobile loop that extends above the ring surface and is also required for activity. The 28 amino acid C-terminal domain, a unique feature of mammalian ClpP proteins, lies on the periphery of the ring, with its proximal portion forming a loop that extends out from the ring surface. Residues at the start of the C-terminal domain impinge on subunit interfaces within the ring and affect heptamer assembly and stability. We propose that the N-terminal peptide of ClpP is a structural component of the substrate translocation channel and may play an important functional role as well. Published by Elsevier Inc.
\end{abstract}

Keywords: Mitochondrial ClpP; Clp/Hsp100; X-ray crystallography; ATP-dependent protease

\section{Introduction}

ClpXP is a bipartite chaperone/protease machine that catalyzes ATP-dependent protein unfolding and degradation in bacteria and in subcellular compartments of eukaryotes (Gottesman, 2003; Halperin et al., 2001; Kang et al., 2002). ClpXP is an important global regulator in bacterial cells, where it targets specific short-lived proteins for degradation and plays essential roles in developmental changes, survival under starvation conditions, replication

\footnotetext{
${ }^{*}$ Corresponding authors. Fax: +1 3014020450 (M.R. Maurizi); +1 3014807629 (B. Ahvazi).

E-mail addresses: mmaurizi@helix.nih.gov (M.R. Maurizi), ahvazib @mail.nih.gov (B. Ahvazi).
}

of phage and plasmids, and in various other regulatory pathways (Gottesman, 2003). It also acts in protein quality control pathways (Gottesman et al., 1997). ClpXP is present in the chloroplasts of plants and photosynthetic bacteria, where it has essential functions and is required for cell viability (Schelin et al., 2002). In humans, hClpX is encoded on chromosome $15 \mathrm{q} 22.2$ (Corydon et al., 2000) and hClpP is encoded on chromosome 19p13.3 (Bross et al., 1995). Both proteins are targeted to mitochondria, but the specific functions of ClpXP in human mitochondria have not been defined.

$\mathrm{ClpP}$ is a self-compartmentalized protease (Lupas et al., 1997); two rings of seven subunits enclose a hollow chamber containing the proteolytic active sites accessible only through axial channels in each ring (Kessel et al., 
1995; Wang et al., 1997). Substrates are presented to ClpP by ClpX or by the related chaperone, ClpA, each of which recognize specific proteins and target them for degradation. ClpX and ClpA are members of the Clp/ Hsp100 family of molecular chaperones (Schirmer et al., 1996) and belong to the $\mathrm{AAA}^{+}$super-family of ATPases associated with various cellular activities, a diverse class of chaperones that disassemble and otherwise modify macromolecular complexes (Neuwald et al., 1999). ClpX and ClpA form stable hexameric rings in the presence of ATP, and the hexamers co-axially stack on each end of ClpP forming barrel-like holoenzyme complexes (Grimaud et al., 1998; Kang et al., 2002).

Face-to-face stacking of the $\mathrm{ClpP}$ rings encloses a chamber with the 14 active sites inside. Each active site has a classical Ser-His-Asp triad composed of residues from a single subunit (Maurizi et al., 1990a; Wang et al., 1997). In Escherichia coli ClpP, the $k_{\text {cat }}$ for peptide bond cleavage is very high $\left(>10,000 \mathrm{~min}^{-1}\right.$ per tetradecamer) (Thompson and Maurizi, 1994), and cleavage specificity is rather tolerant, although hydrophobic side chains are preferred in the $\mathrm{P} 1$ and in the $\mathrm{P} 1^{\prime}-\mathrm{P} 4^{\prime}$ positions. In the E. coli $\mathrm{ClpP}$ crystal structure, a hydrophobic groove connected the active sites within a heptameric ring (Wang et al., 1997). The hClpP and eClpP sequences align without gaps, displaying $\sim 56 \%$ identical and $\sim 70 \%$ similar amino acids (Bross et al., 1995). $\mathrm{HClpP}$ has a 28 -amino acid C-terminal extension, which is not essential for activity.

$\mathrm{HClpP}$ binds to and forms a functional complex with eClpX, indicating that the critical residues for $\mathrm{ClpX}$ interaction are conserved; however, hClpP does not interact with eClpA (Kang et al., 2002). The mechanism of substrate translocation into $\mathrm{ClpP}$ remains a puzzle. The axial portals of eClpP appear large enough to allow only a single helical polypeptide to pass through unobstructed (Wang et al., 1997). However, a disulfide linked polypeptide chain can also enter the ClpP chamber, suggesting either that the access channel is expandable or that two polypeptides in some more collapsed state can pass through the channel (Burton et al., 2001). Regardless of whether one or two chains pass through the channel, it is clear that polypeptide translocation into $\mathrm{ClpP}$ is unfavorable and requires the expenditure of energy to be accomplished.

The symmetry mismatch between the six membered chaperone rings and the seven-membered ClpP rings has led to a proposal that the rings rotate or reciprocate about each other, possibly providing a vectorial force that screws the extended protein into the ClpP chamber (Beuron et al., 1998). Another possible mechanism is that once an end of the substrate enters the ClpA chamber, ClpX or ClpA reptates along the polypeptide by a series of association/dissociation steps with periodically spaced regions of the exposed polypeptide, extruding the polypeptide out the other end and into the aligned channel of ClpP (Ishikawa et al., 2001). Direct support for either of these mechanisms is lacking. The role of $\mathrm{ClpP}$ in the process is also unclear. While simultaneous active proteolysis is not required for translocation (Ishikawa et al., 2001; Ortega et al., 2000), the possibility remains that interactions between the ends of the substrate and sites on the interior of $\mathrm{ClpP}$ may assist unidirectional movement. As part of our efforts to understand the structure/function relationships in Clp proteolytic complexes, we have determined the crystal structure of human ClpP protein. We find that the first seven $\mathrm{N}$-terminal residues are lodged within the axial channel and are needed for ClpP activity.

\section{Experimental procedures}

\subsection{Human mitochondrial ClpP expression and preparation}

Mature hClpP was expressed and purified as described previously (Kang et al., 2002). HClpP with a 6His C-terminal extension was cloned into pVEX11 (Novagen) and expressed in BL21 (DE3). Cells were induced for $4 \mathrm{~h}$ with isopropyl- $\beta$-D-thiogalactopyranoside, collected by centrifugation, and lysed by sonication in $10 \mathrm{mM}$ Tris-HCl, pH 7.5. The centrifuged lysate was applied to a Talon metal chelating column (Invitrogen), the column was washed with 10 volumes of buffer, and the protein was eluted with $20 \mathrm{mM}$ Tris- $\mathrm{HCl}, \mathrm{pH} 7.5$, containing $50 \mathrm{mM}$ imidazole, $100 \mathrm{mM} \mathrm{NaCl}$, and $0.002 \%$ $\mathrm{NaN}_{3}$. HClpP-containing fractions were pooled, supplemented with $5 \mathrm{mM}$ EDTA, and applied to a Q-Sepharose FF column equilibrated with $20 \mathrm{mM}$ Tris- $\mathrm{HCl}, \mathrm{pH} 7.5$, containing $200 \mathrm{mM} \mathrm{NaCl}, 1 \mathrm{mM}$ EDTA, and $1 \mathrm{mM}$ $\mathrm{NaN}_{3}$. The protein was eluted at $300 \mathrm{mM} \mathrm{NaCl}$ in a linear gradient. Fractions with hClpP were then applied to a Sephacryl S200 gel-filtration column in buffer used for QSepharose. For crystallization, hClpP or hClpP-His was dialyzed against $25 \mathrm{mM}$ Bis-Tris, $\mathrm{pH} 6.5$, containing $3 \mathrm{mM}$ DTT, concentrated to $17 \mathrm{mg} / \mathrm{ml}$ using Microcon YM-10 membranes (Millipore), and set up in crystallization trays.

\subsection{Crystallization and data collection}

Crystals of hClpP were grown at $21{ }^{\circ} \mathrm{C}$ by hanging drop vapor diffusion from solutions containing $100 \mathrm{mM}$ Mes, $\mathrm{pH} 6.5,10 \%$ (v/v) dioxane, and 10\% glycerol, and a range of ammonium sulfate from 1.5 to $1.8 \mathrm{M}$. A ruby-like crystal with dimensions of $0.1 \mathrm{~mm} \times 0.25 \mathrm{~mm} \times$ $0.3 \mathrm{~mm}$ was harvested into mother liquor containing $20 \%$ glycerol as the cryoprotectant, flash-cooled into liquid $\mathrm{N}_{2}$ with a rayon mounting loop (Oxford Cryosystems, Oxford, UK) prior to data collection at $100^{\circ} \mathrm{K}$. Data were collected at the National Synchrotron Light Source, 
Table 1

Crystallographic parameters and data processing statistics

\begin{tabular}{|c|c|}
\hline $\begin{array}{l}\text { Space group } \\
\text { Unit cell parameters }\end{array}$ & $\begin{array}{l}\mathrm{C} 2 \\
a=162.57 \AA \\
b=119.00 \AA \\
c=118.65 \AA \\
\beta=130.16^{\circ}\end{array}$ \\
\hline \multicolumn{2}{|l|}{ Data collection statistics } \\
\hline Resolution range $(\AA)^{\mathrm{a}}$ & $20-2.10(2.17-2.10)$ \\
\hline Completeness $(\%)$ & $99.1(99.9)$ \\
\hline Number of reflections & 338348 \\
\hline Number of unique reflections ${ }^{\mathrm{a}}$ & $100429(10076)$ \\
\hline Overall $I / \sigma(I)$ & $19.03(4.19)$ \\
\hline$R_{\text {merge }}{ }^{\mathrm{a}}$ & $0.059(0.281)$ \\
\hline \multicolumn{2}{|l|}{ Refinement Statistics } \\
\hline Resolution range $(\AA)^{\mathrm{a}}$ & $20-2.10(2.17-2.10)$ \\
\hline Number of reflections used & 90655 \\
\hline Protein atoms & 10040 \\
\hline Number of solvent molecules & 773 \\
\hline$R_{\text {crystal }}^{\mathrm{b}} R_{\text {free }}(\%)^{\mathrm{c}}$ & $22.43 / 26.28$ \\
\hline \multicolumn{2}{|l|}{ Average temperature factors $\left(\AA^{2}\right)$} \\
\hline Protein/solvent & $27.98 / 32.91$ \\
\hline \multicolumn{2}{|l|}{ R.m.s. deviation from ideal values } \\
\hline Bond lengths (̊̊) & 0.006 \\
\hline Bond angle $\left({ }^{\circ}\right)$ & 1.3 \\
\hline Dihedral angles $\left({ }^{\circ}\right)$ & 21.5 \\
\hline Improper angles $\left(^{\circ}\right)$ & 0.84 \\
\hline
\end{tabular}

${ }^{\mathrm{a}}$ In parenthesis: highest resolution shell.

${ }^{\mathrm{b}} R_{\text {crystal }}=\sum\left|F_{0}(h)-F_{\mathrm{c}}(h)\right| / \sum F_{\mathrm{o}}(h)$, where $F_{0}(h), F_{\mathrm{c}}(h)$ are the observed and calculated structure factor amplitudes for reflection $h$.

${ }^{\mathrm{c}} R_{\text {free }}$ was calculated against $10 \%$ of the complete data set excluded from refinement.

Brookhaven National Laboratories, using beam line X9B equipped with an ADSC Quantum 4 detector. Diffraction data were indexed, processed, scaled, and merged using the HKL2000 suite of program (Otwinowski and Minor, 1997). The data collection statistics are summarized in Table 1.

\subsection{Structure determination and refinement}

The structure of hClpP was solved by molecular replacement using AmoRe (Navaza and Saludjian, 1997) with E. coli ClpP (PDB 1TYF (Wang et al., 1997)) as a search model and resolution ranges of $10-3.5 \AA$ for the rotation search and $10-3.0 \AA$ for the translation search. There was one solution to both the rotation and translation searches, which gave rise to one molecule within each asymmetric unit in the crystal form in space group C2. Rigid-body refinement of the polyalanine model using CNS (Brunger et al., 1998), followed by simulated annealing applying strict 7-fold non-crystallographic symmetry (NCS) ${ }^{1}$ constraints (Kleywegt, 1996) to $3.0 \AA$ resolution, improved the solu-

\footnotetext{
${ }^{1}$ Abbreviations used: NCS, non-crystallographic symmetry; r.m.s., root mean square; ATP $\gamma \mathrm{S}$, adenosine-5'-(3-thio) triphosphate; SDSPAGE, sodium dodecyl sulfate-polyacrylamide gel electrophoresis.
}

tion to an $R$ factor of $39.8 \%$. Initial phases were improved using solvent flattening (CCP4, 1994), and 7-fold, restrained NCS averaging (Kleywegt and Read, 1997). The polyalanine model was converted to the hclpP sequence using the program $\mathrm{O}$ (Jones et al., 1991). For final refinement, the NCS restraints were released. After many cycles of manual model rebuilding into a SIGMAA-weighted $3\left|F_{\mathrm{o}}\right|-2\left|F_{\mathrm{c}}\right|$ electron density map, the refinement converged at an $R$-factor of $22.52 \%$ applying all low-resolution data sets from $20 \AA$ resolution as bulk solvent correction. Water molecules were included in the model based on hydrogen bonding and the presence of reproducible peaks in electron density maps based on their effects on the free $R$-value. To avoid over fitting of the model, each step of the rebuilding procedure was monitored using the free $R$-factor and a residue real space correlation coefficient as a guide. The quality of the structure was analyzed with the programs PROCHECK (Laskowski et al., 1992) and CNS (Brunger et al., 1998). The figures were generated with Molscript (Kraulis, 1991), and Raster3D (Merritt and Bacon, 1997). The molecular surface property was characterized for topography and electrostatic potential distribution using the program, GRASP (Nicholls et al., 1991).

\subsection{Alternative modeling of eClpP $\mathrm{N}$-terminus}

A $2\left|F_{\mathrm{o}}\right|-\left|F_{\mathrm{c}}\right|$ electron density map for eClpP was calculated based on the deposited structure factors (PDB entry 1TYFP) using the Uppsala University Electron Density Server. Residues 1-7 were modeled into a continuous patch of electron density located within the region that forms the axial channel in the heptamer. The density following residue 7 was broken and ambiguous up to the point at which the continuous chain from residue 19 onward was clearly defined.

\subsection{Generation of hClpP mutants}

Mutations were introduced into the hClpP gene cloned in the T7 promoter driven vector pVEX11. PCR products were obtained using a top strand primer with a start codon at an $N d e I$ restriction site followed by sequences encoding the desired substitution or deletion mutations followed by a 24-base matched priming sequence; the bottom strand primer was hclpPhis_b, which adds a 6-His tag to the C-terminus of hClpP (Kang et al., 2002). PCR products were cut with NdeI and HindIII and cloned into pVEX11. All mutant clones were confirmed by DNA sequencing.

\subsection{Enzymatic assays and other biochemical methods}

Purified hClpP and mutant derivatives were assayed for protease activity using either $\alpha$-casein as substrate 
and $\mathrm{hClpX}$ as the activator or phage $\lambda \mathrm{O}$ protein as a substrate and $\mathrm{eClpX}$ as the activator. Assay conditions were described previously (Kang et al., 2002). Peptidase activity was measured using the decapeptide, FAPHMALVPV, which is cleaved between methionine and alanine yielding products that were separated by reverse phase chromatography and detected and quantitated on a Hewlett-Packard 1090 Chem-Station as described (Thompson and Maurizi, 1994). N-terminal sequencing was performed on proteins blotted to PDVF membranes using an Applied Biosystems 431A peptide sequencer.

Assembly of hClpXP complexes was monitored by ultrafiltration using UFC300-MC membranes (Millipore). $\mathrm{HClpX}$ and different hClpP mutant proteins were added to a final volume of $50 \mu \mathrm{l}$ of $50 \mathrm{mM}$ Hepes, $\mathrm{pH}$ 7.5, $0.1 \mathrm{M} \mathrm{KCl}$, and $10 \%$ (v/v) glycerol containing $1 \mathrm{mM}$ ATP $\gamma \mathrm{S}$ and $10 \mathrm{mM} \mathrm{MgCl} 2$. A portion of the mixture was taken for reference and the remainder was subjected to centrifugation according to the manufacturer's recommendation. Equivalent aliquots of the initial mixture, the filtrate, and the fraction retained by the membrane were analyzed by SDS-PAGE, and the amounts $\mathrm{hClpX}$ and hClpP mutant proteins were estimated by scanning and densitometry following staining with Coomassie blue. The membranes were reversed and retained proteins were flushed back off the membranes with buffer. When the proteins were present alone, $\sim 20 \%$ of the $\mathrm{hClpX}$ and $\sim 5 \%$ of most of the hClpP mutant proteins were retained by the membranes, with the exception of hClpP-N $\Delta 8-15$ of which $\sim 50 \%$ was retained.

\section{Results and discussion}

\subsection{Structure determination}

The mature form of hClpP was expressed in E. coli cells by initiating translation at amino acid 57 , the predicted site for processing of this mitochondrial protein (Kang et al., 2002). The protein folded and assembled into a seven-membered ring in vivo, and the isolated protease interacted with purified hClpX and expressed activity. The presence of the intact protein, residues 2 221 after removal of the N-terminal methionine $\left(M_{\mathrm{r}}\right.$ 24,781), was confirmed by mass spectroscopy, although a small amount of hClpP retained the N-terminal methionine. Crystals of hClpP diffracted to a resolution of 2.1 $\mathrm{A}$. HClpP is identical to eClpP in 108 of the 193 overlapping amino acids residues, with an additional 45 moderately conserved positions (Fig. 1A), which enabled us to use initial phases from the previously determined crystal structure of eClpP (Wang et al., 1997) to calculate the initial electron density maps of hClpP. After refinement, the density maps were of good quality (Fig. 1B), allowing nearly complete tracing of the main chain and side chains of residues from 2 to 193 in all se-

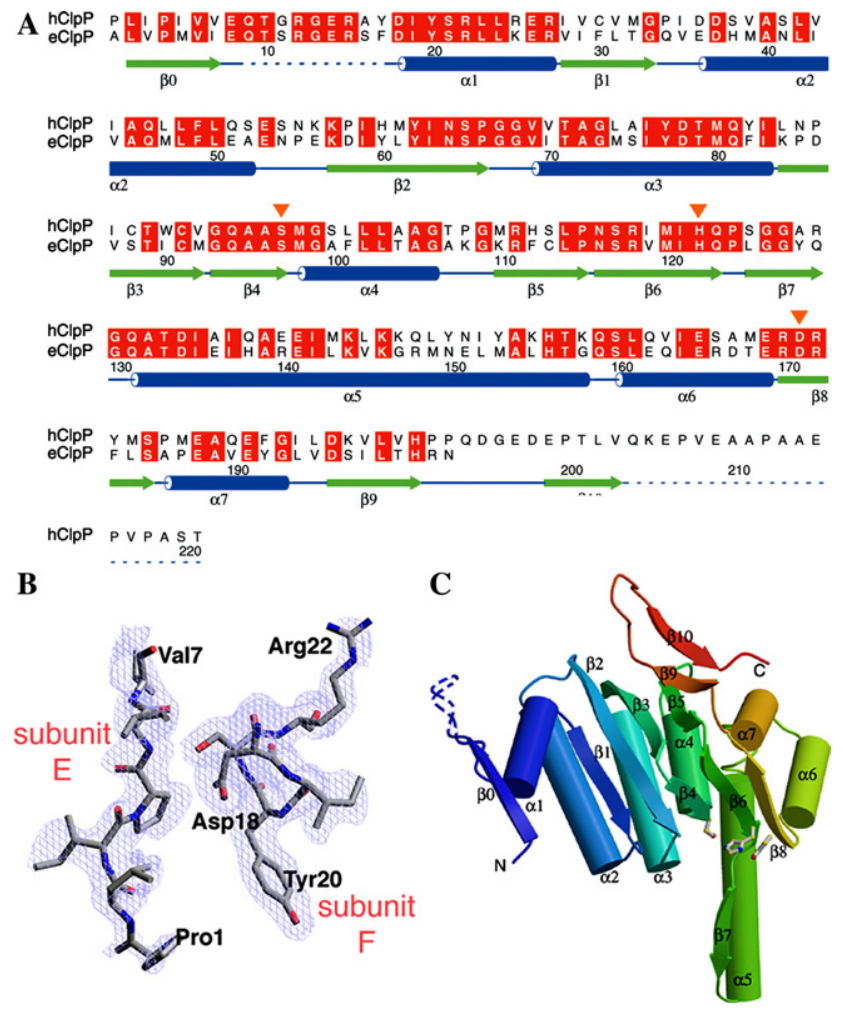

Fig. 1. Subunit structure of hClpP. (A) Sequence alignment of hClpP and eClpP. Sequence alignments are shown with identical and conserved residues boxed. The members of the catalytic triad Ser97, His122 and Asp171 are shown by an inverted triangle. Secondary structure assignments were obtained by the program O (Jones et al., 1991); sequence graphics were made with ALSCRIPT (Barton, 1993). (B) Portion of the $3\left|F_{\mathrm{o}}\right|-2\left|F_{\mathrm{c}}\right|$ electron density of subunit $\mathrm{E}$ in the region of the $\mathrm{N}$-terminal peptide and its interface with the adjacent subunit F. (C) Ribbon tracing of one hClpP subunit showing its $\alpha / \beta$ motif, two, slightly offset, perpendicular $\beta$ sheets, and the long $\alpha / \beta$ handle region projecting from the globular domain. The polypeptide chain is shown in rainbow colors from blue at the $\mathrm{N}$-terminus to red at the C-terminus. The residues of catalytic triad: Ser97, His122, and Asp171 are shown as ball and stick models. The 7-fold axis runs vertically.

ven subunits (Fig. 1C). Only residues $8-16$ had poorly defined densities. In three hClpP subunits, portions of a C-terminal extension, unique to mammalian ClpP, were traced between residues 194 and 199 (subunit D) and between 194 and 205 (subunits F and G). In the crystal, the asymmetric unit consists of one heptameric ring with overall dimensions of $50 \AA$ in height and $90 \AA$ in diameter. The refinement details are shown in Table 1.

The overall fold of hClpP is very similar to that of eClpP (Fig. 2A). The C $\alpha$ backbone of residues 18-190 of one monomer of eClpP superimpose onto a monomer of hClpP with an r.m.s. deviation of only $0.63 \AA$. Each monomer folds into a wedge-shaped globular domain attached to a $28-\AA$ long $\alpha / \beta$ arm consisting of tightly bonded $\beta 9$ and $\alpha 5$ (Figs. $1 \mathrm{C}$ and $2 \mathrm{~A}$ and $\mathrm{B}$ ). The globular domain has two perpendicular $\beta$ sheets made up of 

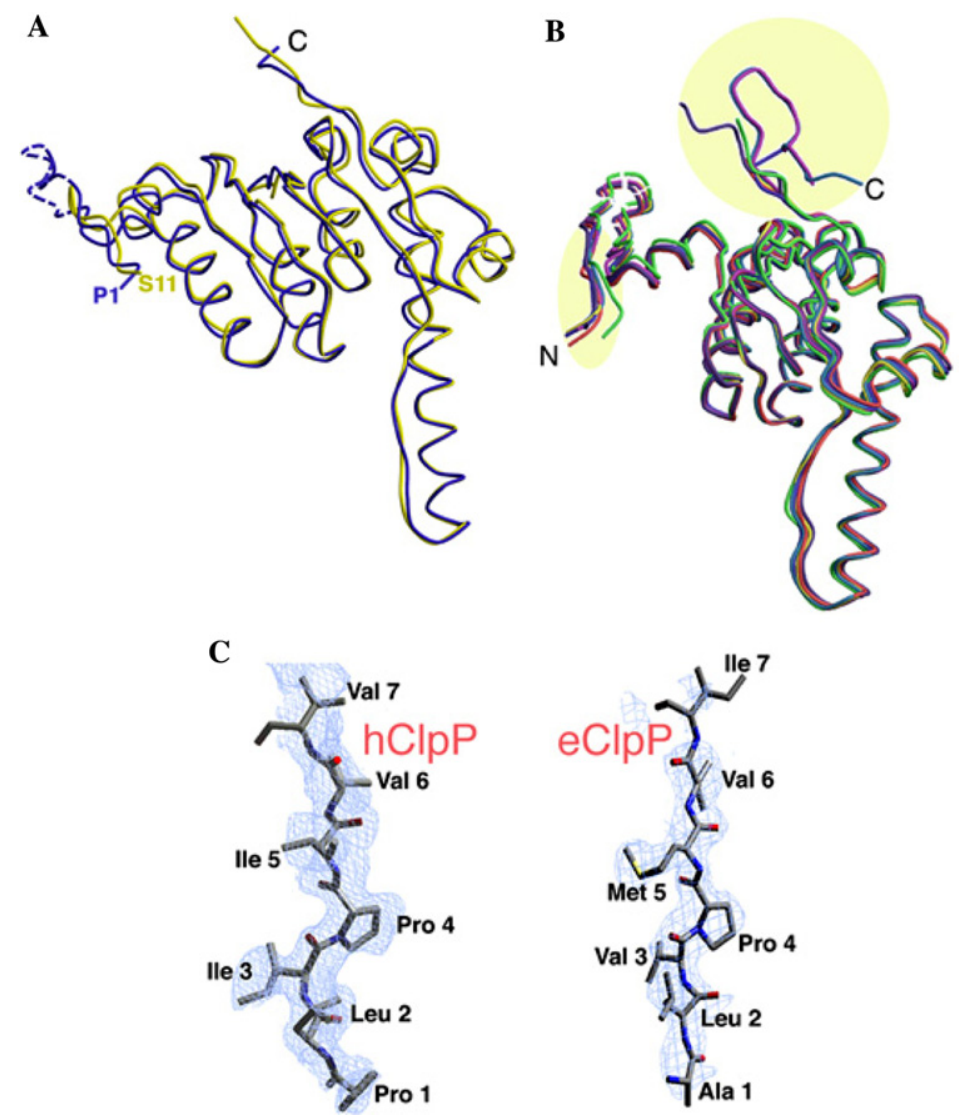

Fig. 2. (A) Superposition of C $\alpha$ backbones for residues 1-193 of hClpP and residues 11-193 of eClp (PDB entry 1TYF) (Wang et al., 1997). The first 10 residues of eClpP were not shown in the model published and were proposed to be highly mobile. In the model of hClpP proposed here, residues 1-7 occupy the density assigned to eClpP residues 1-7. Residues 8-17 of hClpP, for which only broken density was visible, are believed to be highly mobile and are shown as a dashed line. (B) Tracing of the $\mathrm{C} \alpha$ backbones in all seven subunits of hClpP. The N-and C-terminal regions, which show the most mobility and incomplete electron density, are highlighted in yellow. (C) The $3\left|F_{\mathrm{o}}\right|-2\left|F_{\mathrm{c}}\right|$ electron density map contoured at $1 \sigma$ level of the $\mathrm{N}$-terminal region of $\mathrm{hClpP}$ determined in this study compared to the $2\left|F_{\mathrm{o}}\right|-\left|F_{\mathrm{c}}\right|$ electron density contoured at $1 \sigma$ in the same region in the structure of eClpP. EClpP density was calculated from the structure factors (PDB entry 1TYFP) using the Uppsala Electron Density Server (http:// eds.bmc.uu.se/eds). We found that residues 1-7 from each protein could be modeled into their respective electron densities, suggesting that the $\mathrm{N}$-termini of the two proteins adopt the same conformation.

parallel strands $1,3,5,7$, and 11 ( $\beta$ sheet 1$)$ and parallel strands $2,4,6$, and 8 , and anti-parallel strand 10 ( $\beta$ sheet 2 ), respectively, with helices $\alpha 2-\alpha 4, \alpha 6$, and $\alpha 7$ tucked into the fold of the $\beta$ sheets. The subunit interfaces within the heptamer are formed by packing of helices $\alpha 2$ and $\alpha 3$ of one subunit against $\beta$ sheet 1 of the next subunit. Additional contacts include $\alpha 2$ with $\alpha 1$ of the next and $\alpha 5$ with $\beta$ sheet 2 and $\alpha 7$ of the next subunit. In addition to these stable interactions, $\alpha 2$ and the proximal part of $\alpha 1$ make contact with the $\beta$ strand formed by residues $1-$ 8 , which runs from the inside out parallel to the axial channel.

\subsection{Crown and channel-forming role of the $N$-terminus of $h C l p P$}

The hClpP structural model presented here has a unique feature that distinguishes it from the previously determined eClpP. In hClpP, the first seven $\mathrm{N}$-terminal residues are anchored in place as strand $\beta 0^{2}$ (Fig. 2C), and residues $8-16$ are not clearly visible, although broken density was observed in this region for most of the subunits. The orientation of $\beta 0$, inside to outside, places residues $8-16$ on the outer surface of the heptamer. Because these nine residues must adopt a configuration that rejoins the main chain at position 17 and the glycine residues at positions 11 and 13 would favor a bend in the polypeptide backbone, we have modeled residues $8-16$ as a loop creating a crown for the central portal (Fig. $3 \mathrm{~A}$, right). The lack of assignable density for residues $8-16$ indicates that this loop is mobile. The $\mathrm{N}$-terminal $\beta 0$ fits into a hydrophobic cleft formed by helix $\alpha 1$,

\footnotetext{
${ }^{2}$ The initial beta strand, which was not visible in the eClpP crystal structure, is labeled $\beta 0$ to maintain consistency in the naming of the secondary structure elements in $\mathrm{hClpP}$ and eClpP. The coordinate of hClpP has been deposited in the Protein Data Bank with Accession No. 1TG6.
} 
A

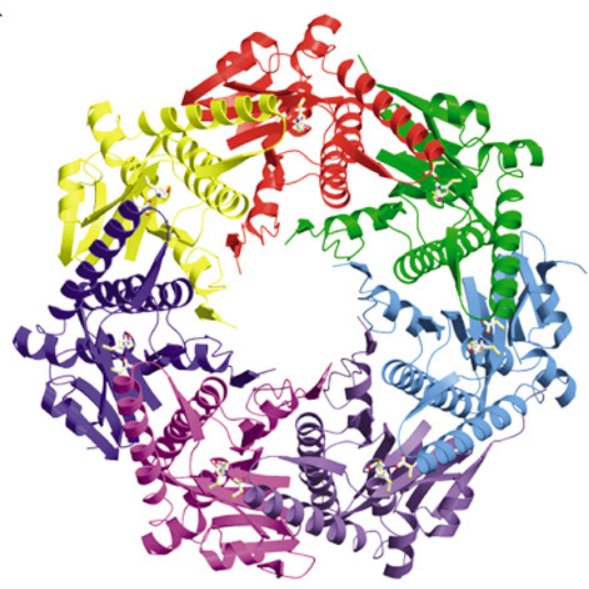

B

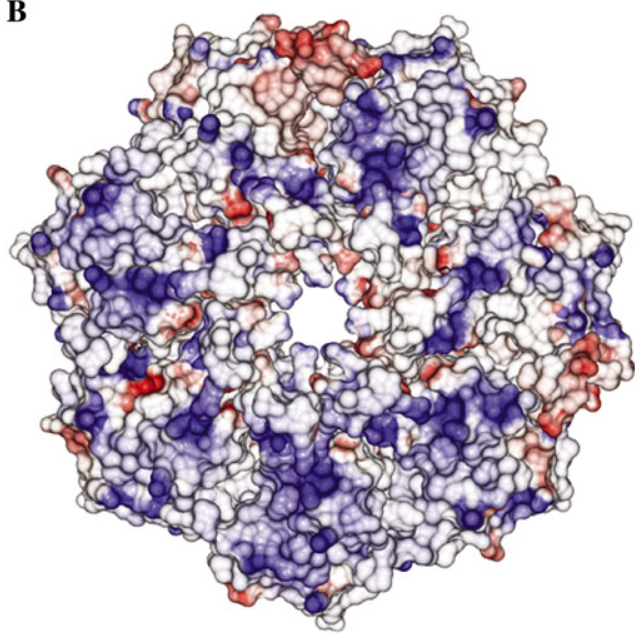

C

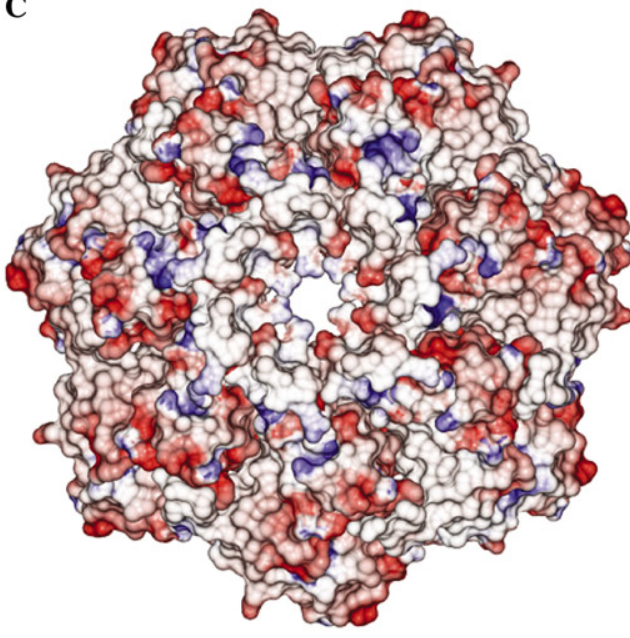

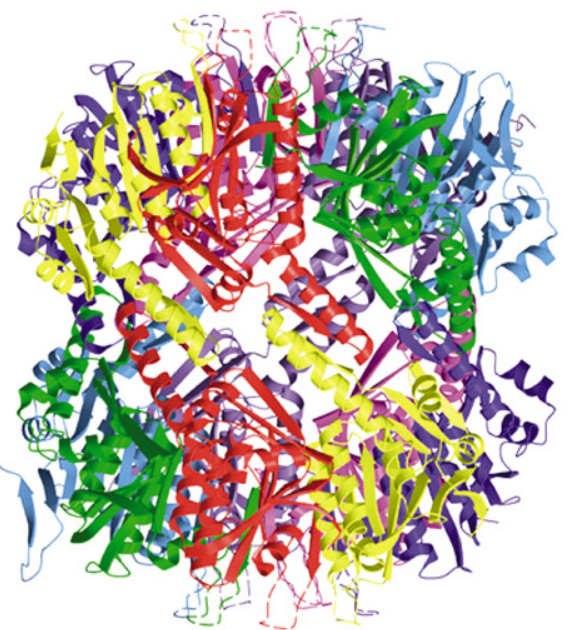
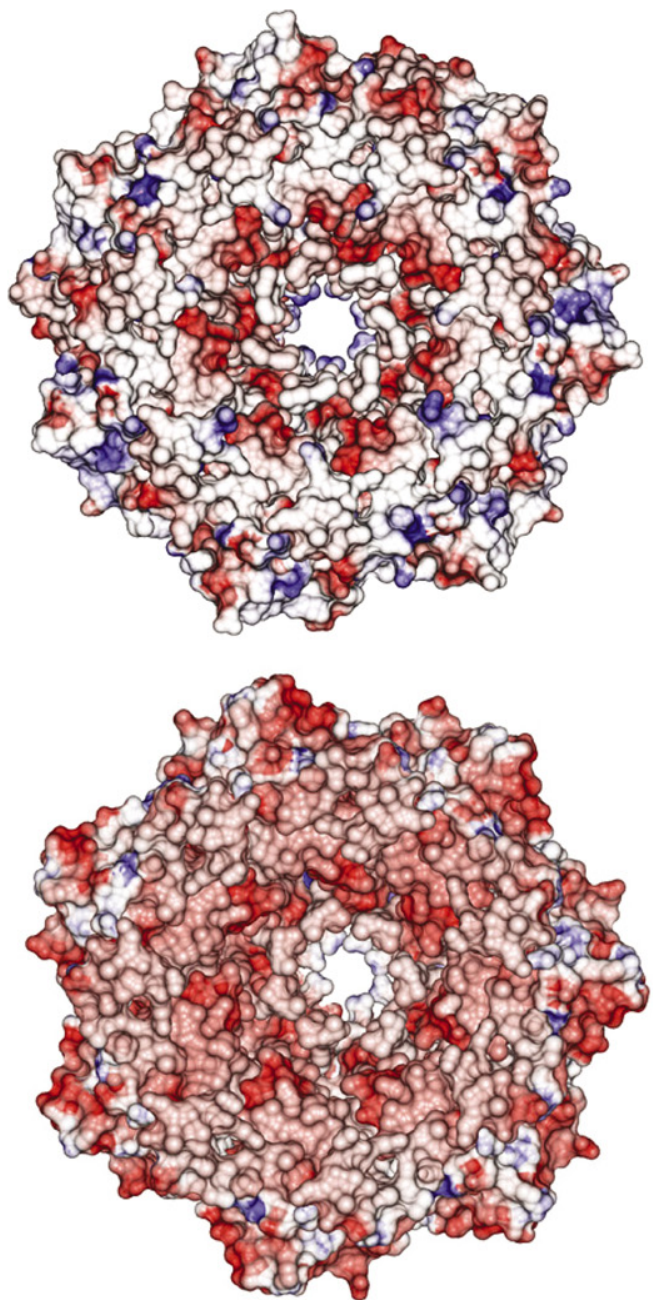

Fig. 3. (A) Ribbon representations of the hClpP viewed down the 7-fold axis. Left, heptamer in the asymmetric unit with the apical ring surface, which forms the interface with the chaperone components, facing out. Right, the hClpP tetradecamer composed of two heptameric, rings bound faceto-face. The ring interface is made by the intercalating $\alpha / \beta$ arms, which also form the lateral walls of the degradation chamber. The $N$-terminal loops protruding from each apical surface have been modeled to show their prominent position in the center of the chaperone-binding surface. (B) Electrostatic potential surfaces of $\mathrm{hClpP}$ are shown with negatively charged regions in red and positively charged regions in blue from -10.0 to $+10.0 \mathrm{kT}$, respectively. Left, apical surface, which interacts with ClpX. Right, a rotation of the, heptamer by $180^{\circ}$ producing a view looking into the degradation chamber from the vantage point of the interface between the ClpP rings in the tetradecamer. (C) Electrostatic potential surfaces of eClpP shown in the same orientations. 
$\mathbf{A}$

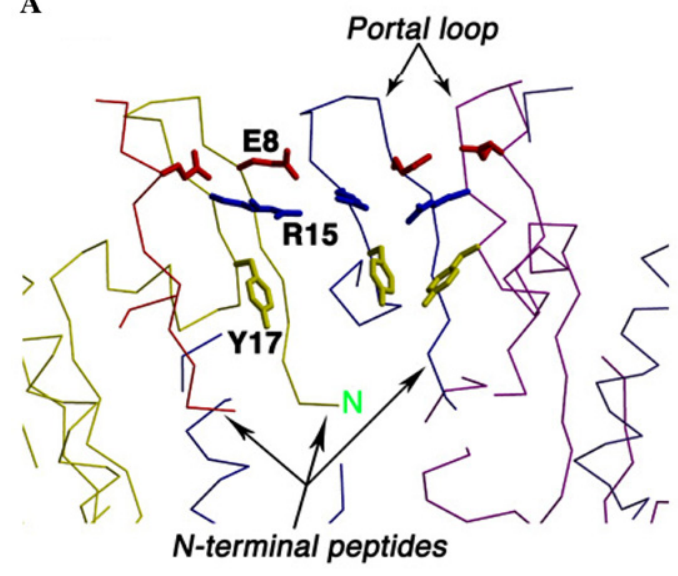

B

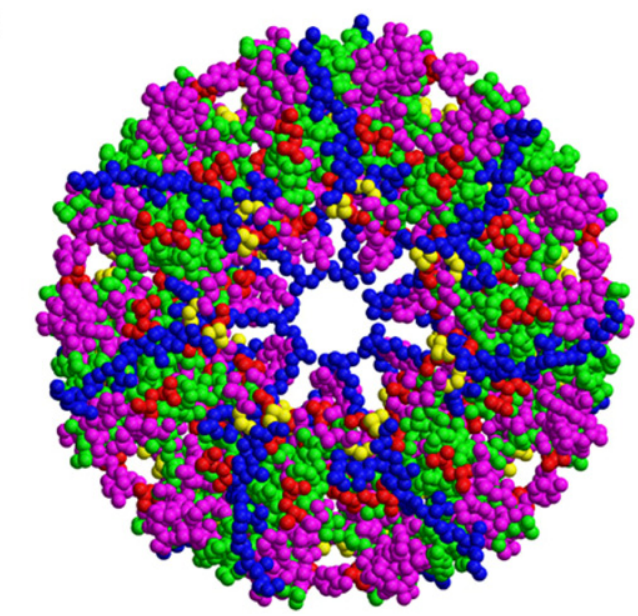

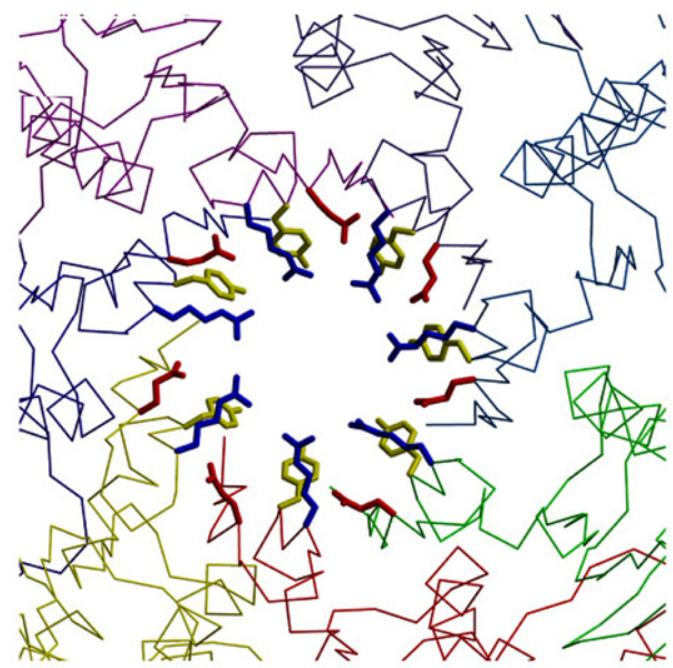

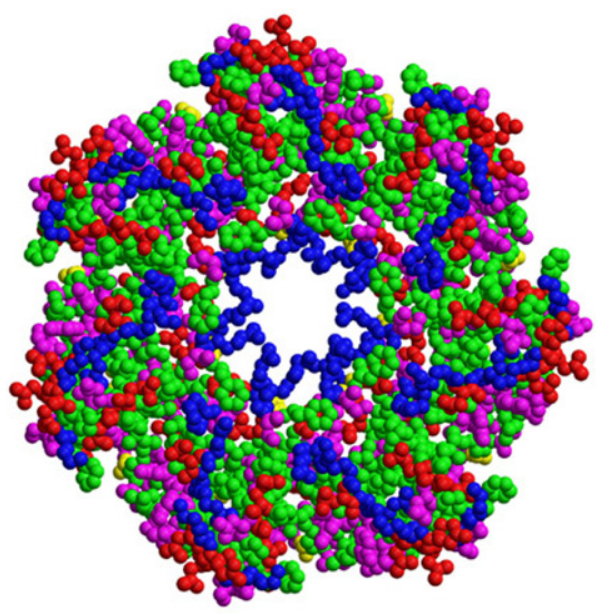

Fig. 4. (A) A view of the solvent accessible axial portal in the heptameric ring showing the positions of the N-terminal peptides lining the wall of the access channel (left). The position of the N-terminal peptide is stabilized by a hydrogen bond from the side chain of Glu 8 to Gly 13 of the adjacent subunit. The side chains of Arg15 and Tyr17 point towards the center like spokes of a wheel (right). (B) CPK representations of hClpP and eClpP apical ring surfaces. The model of eClpP is shown with the N-terminal peptide bound within the axial channel as determined in this study (see Fig. 2). The hydrophobic residues (Tyr, Phe, Try, Leu, Ile, Val, Pro, and Ala) are colored green, positively charged residues (Lys and Arg) blue, and negatively charged residues (Asp and Glu) red. All other residues are colored magenta, except Gly, which is yellow. The surface of hClpP is overall less hydrophobic than that of eClpP.

strand $\beta 1$, and helix $\alpha 3$ of the adjacent subunit, where it could provide additional stability to the heptamer. The side chains in residues 1-7 could not all be modeled into the N-terminal density, but the densities for Ile3, Pro4, and Ile5 side chains were clear (Fig. 2C). No N-terminal methionine density was visible, suggesting that the unprocessed $\mathrm{N}$-terminal methionine in $10-15 \%$ of the subunits was randomly distributed. The $\mathrm{C} \alpha$ backbone atoms of the $\mathrm{N}$-terminal peptides from all seven subunits aligned with each other (Fig. 2B). The backbone carbon atoms superimpose with an r.m.s. deviation of $<1.0 \AA$, although the density for individual side chains are oriented differently in different subunits (data not shown). Alternative models in which residues $12-18$ were fit into the density gave consistently poorer refinement statistics for all of the subunits. In a separate study, we have solved the structures of hClpP bearing a C-terminal 6histidine tag (at $2.2 \AA$ resolution) as well as hClpP modified with a peptide chloromethyl ketone (at $2.4 \AA$ resolution), both of which resulted in better refinement statistics when the $\mathrm{N}$-terminal peptides were modeled as shown here into similar patches of electron density (data not shown).

In contrast, the published model of eClpP (Wang et al., 1997) had the first 10 residues disordered, and a short loop beginning with residue 11 was modeled prior to the helix $\alpha 1$ (Fig. 2A). However, we reexamined the $2\left|F_{\mathrm{o}}\right|-\left|F_{\mathrm{c}}\right|$ electron density map of eClpP and found that a somewhat better fit can be obtained by modeling the N-terminus similarly to the way we propose for the hClpP N-terminus (Fig. 2C). Residues Val3, Pro4, and Met5 of eClpP adopt virtually the same orientation as 
A

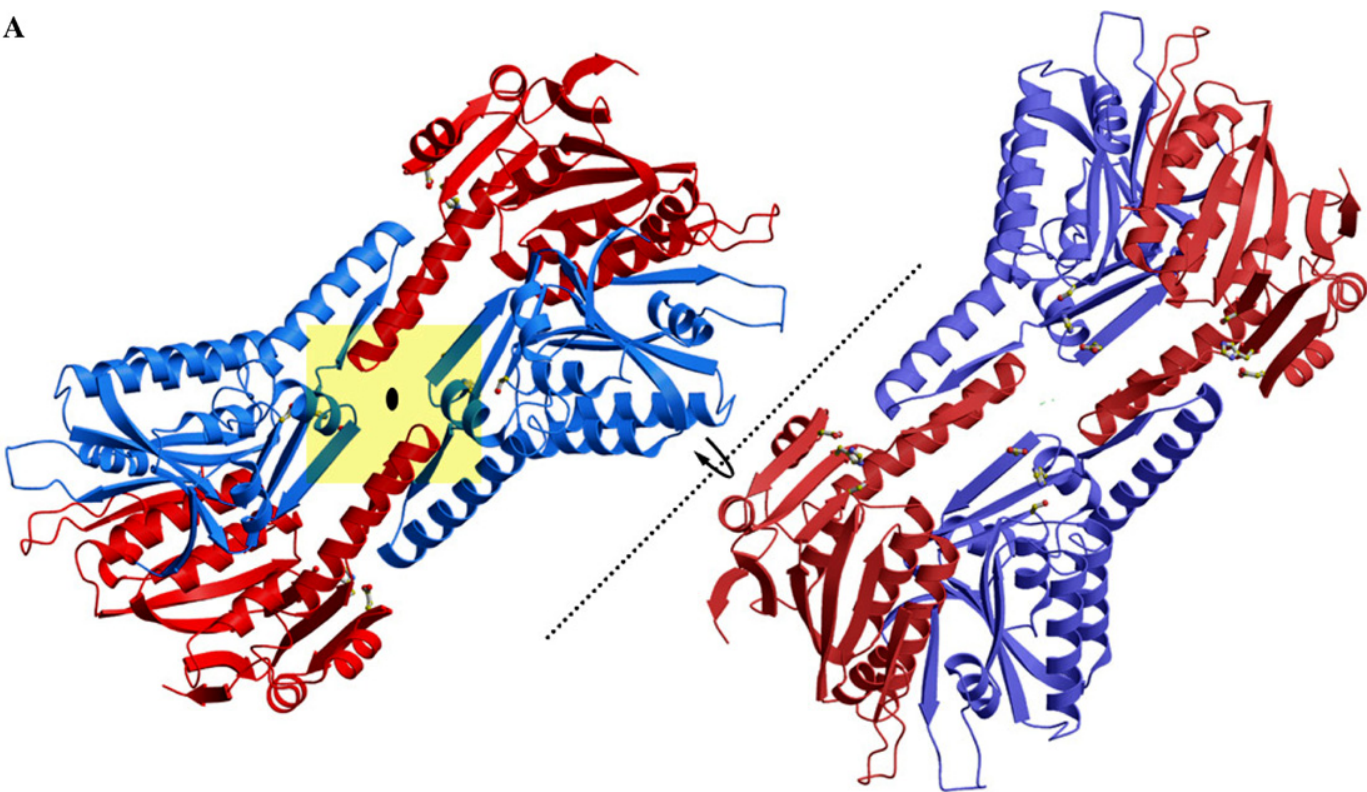

B

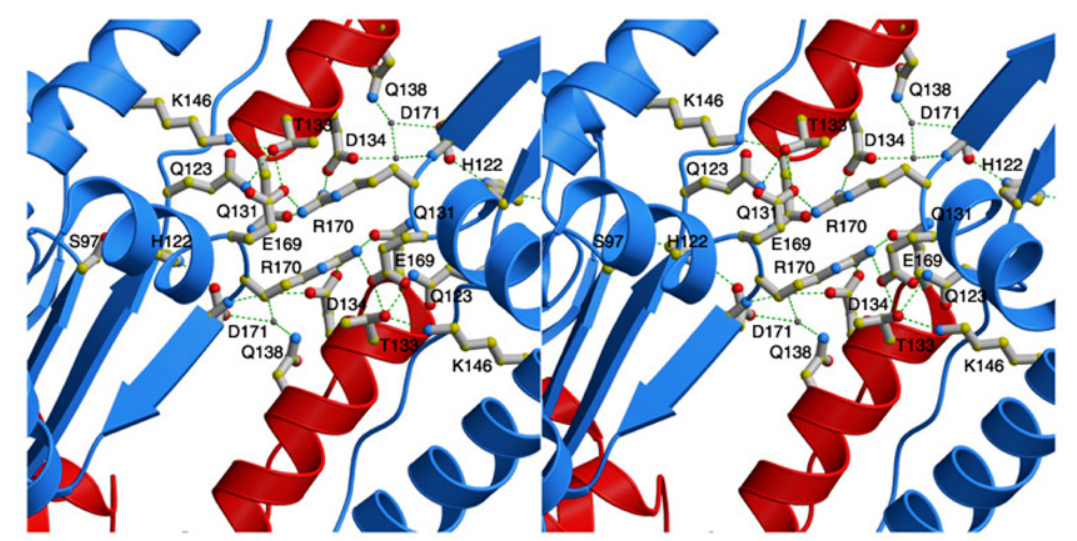

Fig. 5. (A) The dimerization interface of the two subunits across the heptameric ring viewed from the outside of the annulus. Two NCS-related subunits in hClpP are isologously bonded subunits across the tetradecamer interface. An anti-parallel sheet is formed on the inside of the ring by interaction of strand $\beta 7$ (equivalent to $\beta 9$ in eClpP) from each subunit. Side chains from helices $\alpha 5$ from the two apposed subunits make symmetrical hydrophobic contacts on the outside of the annulus. The location of the 2-fold axis of symmetry relating the two subunits of intra ring of heptamers is also shown. (B) The loop between $\beta 7$ and $\alpha 6$ participates in an intra-ring web of hydrogen bonding contacts involving residues at the base of the active site cleft and close to the catalytic Asp171, suggesting that assembly of the tetradecamer could influence the configuration of the catalytic triad.

residues Ile3, Pro4, and Ile5 of hClpP (Fig. 2C). Thus, both species of $\mathrm{ClpP}$ appear to have an $\mathrm{N}$-terminal peptide located within the substrate access channel and have a disordered or mobile loop composed of residues 8-16.

\subsection{Tetradecamer structure}

In our crystals, two heptamers related by a $180^{\circ}$ rotation about a 2-fold symmetry axis were joined to form a tetradecamer. Because the molecular and crystallographic symmetry axes coincided, the asymmetric unit contained a single heptamer. The tetradecamer consists of the two domed-shaped heptamers with their arms interlocked creating a closed cylindrical particle pinched in the middle (Fig. 3A, right). $\beta 9$ is tandemly aligned with $\beta 8$ from $\beta$ sheet 2 ; following a turn, the 23 -amino acid long helix $\alpha 5$ returns, and the C-terminal end is embedded in the globular domain. As in eClpP, the tetradecamer encloses an aqueous chamber $58 \AA$ in diameter (Figs. 3B and $\mathrm{C}$ ). The $\mathrm{N}$-terminus and strand $\beta 0$ are located within the axial channel (Figs. 3A and $4 \mathrm{~A})$. The $\mathrm{C}$-terminal domains ( $\beta 10$ and $\beta 11)$ are located on the distal surface on the outer rim of the heptamer extending across neighboring monomers. The seven monomers superimpose well with the exception of variability in the location of the C-terminus, which may have been affected by crystal lattice contacts (data not shown).

The N-termini ( $\beta 0, \alpha 1$, and the intervening loop) form the rim of the central portal that extends above the surface of the heptamer (Fig. 3A), and a funnelshaped channel provides access to the inner proteolytic 


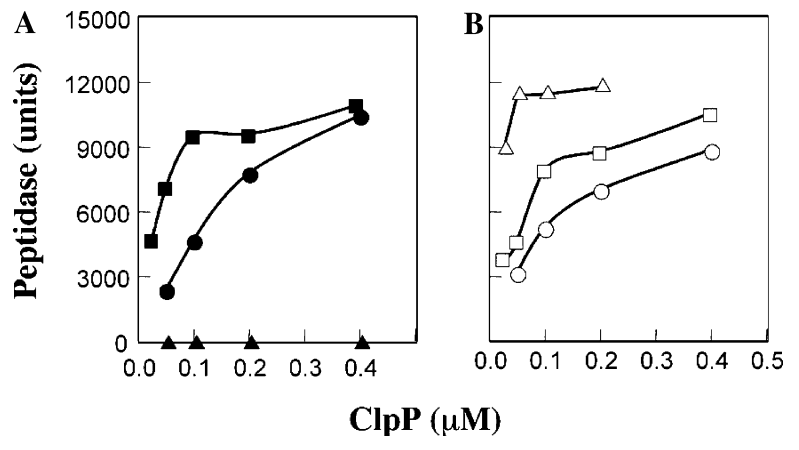

Fig. 6. Removal of the C-terminus of hClpP improves interaction with hClpX. Formation of complexes between hClpP and either hClpX or eClpX was measured by activation of peptide degradation in the presence of $\mathrm{ATP} \gamma \mathrm{S}$ (Thompson and Maurizi, 1994). Assays were conducted with $0.1 \mu \mathrm{M}$ hClpX (A) or $0.1 \mu \mathrm{M}$ eClpX (B) and increasing amounts of hClpP (circles), hClpP- $\Delta \mathrm{C}$ (squares), or eClpP (triangles), as shown.

chamber. The solvent accessible diameter of the axial portal is $\sim 25 \AA$ measured between $\mathrm{C} \alpha$ atoms but is made narrower $(12-20 \AA)$ by the side chains of Glu8, Arg15, and Tyr17 pointing towards the central axis like the spines of a fish trap (Fig. 4A). The size of the channel is comparable to substrate channels in $\operatorname{HslV}(\sim 20 \AA)$ and the $\beta$ subunits of the $20 \mathrm{~S}$ proteasome $(\sim 27 \AA)$ (Bochtler et al., 1997; Lowe et al., 1995). These features of the channel may be general for all ClpP proteins, because the eClpP model we constructed with the N-terminus within the axial channel shows a similar axial pore (Fig. 4B). These polar and charged residues may affect the passage of substrates. The crown of the cylinder formed by contacts between the wedge domains is thick, whereas the walls of the cylindrical chamber formed by interlocking of the arms are thin by comparison. The two rings are held together by extensive hydrogen bonding along the backbones of the anti-parallel $\beta 9$ strands from apposing subunits (Figs. 5A and B). The spaces between the strands are filled by the $\alpha 5$ helices, which interact with the side chains of $\beta 9$ from the apposing subunit by way of numerous electrostatic and hydrogen bonding interactions.

\subsection{Surface features of $h \mathrm{ClpP}$}

Within $\sim 35 \AA$ of the central axes, the apical surfaces of the hClpP and eClpP tetradecamers have similar characteristics, with the exception of possible differences near the N-terminal loops as discussed below. Most of the non-conservative amino acid changes are on the outer edge of the apical surface and along the lateral surface of the tetradecamer (Fig. 4B). Of the apically exposed residues, the most notable changes introduce hydrophilic residues into the radial hydrophobic groove postulated to be a potential docking site for the chaperone components in eClpP (Wang et al., 1997). The
hClpP residues, Asn55, His60, and His112, reduce hydrophobicity and, together with a charge inversion produced by the Glu56Lys substitution, create a positively charged segment in the distal portion of the docking groove. In other experiments, we have found that chemical modification of this region interferes with ClpX-dependent activities, suggesting that it is involved in interaction with ClpX, (S.G.K. and M.R.M., in preparation).

There are 12 hydrophobic residues (Pro58, Leu84, Pro86, Try90, Pro108, Met110, Ala136, Val163, Phe182, Val190, Pro192, and Pro193) introduced onto the lateral surface of the hClpP tetradecamer compared to eClpP. The shift to a more hydrophobic surface is dominated by proline residues that cluster in two locations on the lateral surface, suggesting that hClpP may engage in interactions with other factors within the mitochondria. There is a slight shift towards a more positive surface potential, particularly nearer to the apical surface, where basic residues were introduced (Gly147Lys, Leu155Lys, and Gly158Lys), whereas the equatorial region near the interface of the heptamers have a greater negative surface potential due to the loss of basic residues (His138Gln, Arg140Glu, and Arg166Ser). The ClpX and $\mathrm{ClpA}$ are larger in diameter than $\mathrm{ClpP}$, and the residues on the lateral surface, particularly close to the apical surface, could interact with the chaperone to stabilize the complex, as is seen in the HslUV (ClpYQ) complex (Sousa et al., 2000). One particular change, Glu56Lys, which places a positive charge just at the outer edge of the apical domain is noteworthy in this respect and might be responsible for the weaker interaction of hClpP with eClpX. These considerations are under further investigation.

\subsection{Effects of the C-terminal domains on structure and assembly}

HClpP has an additional 28 residues at the C-terminus. The C-termini in different subunits appear to be flexible and variable, as they appear in one conformation in two monomers involved in a lattice contact, a second conformation in two adjacent monomers, and disordered in three monomers. None of the C-terminal domains was visible in its entirety. In subunits $\mathrm{E}$ and $\mathrm{G}$, residues 194-203 form a $\beta$-hairpin that extends $\sim 10 \AA$ above the surface on the outer edge of the heptamer ring (Figs. 2B and 3B). Positions 192 and 193, which constitute the $\mathrm{C}$-terminus of $\mathrm{eClpP}$, play pivotal roles in stabilizing the C-terminus. In hClpP, Pro192 makes close hydrophobic contacts with Tyr82 and Leu84 of the adjacent subunit, while Pro193 forms part of an intra-subunit hydrophobic core at the base of the C-terminal loop. In eClpP, the terminal residues His 191 and Asn 193 make hydrogen bonding interactions with backbone atoms of the adjacent subunit and the side 
chain of Arg192 makes an intrasubunit hydrogen bond. When hClpP was expressed without its C-terminal extension, the protein was structurally unstable with prolines in the terminal positions; however, replacing the prolines with the eClpP residues (Arg and Asn) produced a stable active heptameric protein. Thus, assembly of hClpP heptamers may be influenced by factors that affect the conformation of the C-terminal domain.

The C-terminal domain also influences anchoring of the components of the holoenzyme complex. Titrations of hClpX and eClpX showed that hClpP- $\Delta \mathrm{C}$ has a higher affinity for both hClpX and eClpX than does wild type hClpP (Figs. 6A and B). Since no other differences in activities were observed between hClpP and hClpP$\Delta \mathrm{C}$, these data imply that the $\mathrm{C}$-terminal domains may sterically interfere with interaction between $\mathrm{hClpP}$ and $\mathrm{hClpX}$ or that the presence of the C-terminal region affects the conformation of the surface that interacts with ClpX. The proline-rich C-terminal domain may be a docking site for endogenous mitochondrial factors that could influence its effect on assembly of the holoenzyme complexes; however, no structural homologs of the $\mathrm{C}$-terminal domain have yet been identified.

\subsection{Enzymatic importance of the $N$-terminus of hClpP}

To test the prediction that the N-terminus plays an essential role in hClpP activity, we created a number of deletion and substitution mutations within the first 15 amino acids. The non-interacting N-terminal Pro1 could be deleted or replaced without significant effect on the activity of hClpP (Table 2). However, deletions of residues 2-5 and deletions of 10 or 15 amino terminal residues led to severe impairment of activity, with deletion of more than two residues leading to virtually inactive hClpP. Loss of activity in the deletion mutants was not due to problems with assembly of the hClpP, which showed behavior identical to that of wild type during gel filtration and native gel electrophoresis (data not shown). One mutation, the Pro4Ala, did lead to structural instability of hClpP; this mutant aggregated and was highly susceptible to proteolytic digestion by trypsin and chymotrypsin in vitro (data not shown), suggesting that the Pro4 residue contributes to the structural integrity of this region of the protein. In the crystal structure, Pro4 faces a hydrophobic pocket on the adjacent subunit (Fig. 1B) and likely helps to anchor the hydrophobic N-terminal peptide within the channel. In comparing $\mathrm{N}$-terminal sequences from $>50 \mathrm{ClpP}$ proteins, a consensus, $\mathrm{x} \Phi \Phi \mathrm{P} \Phi \Phi \Phi \mathrm{E}$, which includes the invariant Pro4, the invariant negatively charged, Glu8, and five hydrophobic residues $(\Phi)$ can be defined. This consensus further points to an important structural or functional role for this portion of ClpP.

Although most of the N-terminally deleted hClpP proteins folded normally, they showed systematic defects in enzymatic activity (Table 2). Mutants deleted beyond Leu2 had low peptidase activity, suggesting either that peptides did not have access to the active sites or that conformational differences at the $\mathrm{N}$-terminus were

Table 2

Activities of hClpP mutants with deletions or substitutions in the N-terminus

\begin{tabular}{|c|c|c|c|c|c|}
\hline \multirow{2}{*}{$\begin{array}{l}\text { Mutants }^{\mathrm{a}} \\
\text { Wild type }\end{array}$} & \multirow{2}{*}{$\begin{array}{l}\text { Heptamer }^{\mathrm{b}} \text { formation } \\
+\end{array}$} & \multicolumn{2}{|c|}{ Peptidase $^{\mathrm{c}} \mathrm{ClpP}$ alone $(+\mathrm{hClpX})$} & \multicolumn{2}{|c|}{$\begin{array}{l}\text { Protein degradation }{ }^{\mathrm{d}} \text { with } \\
\mathrm{hClpX}(+\mathrm{eClpX})\end{array}$} \\
\hline & & 1.0 & $(1.0)$ & 1.0 & $(1.0)$ \\
\hline hClpP-P1A & + & 0.4 & $(1.0)$ & 0.9 & $(1.0)$ \\
\hline hClpP-P1C & + & 0.2 & $(0.13)$ & 0.16 & $(0.2)$ \\
\hline hClpP-N $\Delta 1 \mathrm{~L} 2 \mathrm{~A}$ & + & 0.1 & $(0.06)$ & 0.14 & $(0.13)$ \\
\hline hClpP N $\Delta 2 \mathrm{I} 3 \mathrm{~V}$ & + & N.D. & $(0.06)$ & 0.13 & $(0.09)$ \\
\hline hClpP-N $\Delta 3$ & + & 0 & $(0)$ & 0 & $(0)$ \\
\hline hClpP-N $\Delta 4$ & + & 0 & $(0.01)$ & 0 & $(0.01)$ \\
\hline hClpP-N $\Delta 10$ & + & N.D. & $(0)$ & 0 & $(0)$ \\
\hline hClpP-N $\Delta 15$ & + & N.D. & $(0)$ & 0 & $(0)$ \\
\hline hClpP-P4A & $\mathrm{X}$ & N.D. & $(0)$ & 0 & $(0)$ \\
\hline hClpP-eN7 & + & N.D. & $(0.6)$ & 0.7 & $(0.65)$ \\
\hline hClpP-N $\Delta 8-15$ & + & N.D. & $(0)$ & 0 & (N.D.) \\
\hline hClpP-NHis & + & N.D. & $(0.3)$ & 0.2 & (N.D.) \\
\hline hClpP-N+14 & + & N.D. & $(0.8)$ & 0.5 & (N.D.) \\
\hline hClpP-N+4 & + & N.D. & $(0.9)$ & 0.8 & (N.D.) \\
\hline hClpP-N+2 & + & N.D. & $(1.0)$ & 1.0 & (N.D.) \\
\hline \multicolumn{6}{|c|}{$\begin{array}{l}\text { N.D., not determined. } \\
\text { a The mutants are as follows: ClpP-eN7, first seven amino acids of eClpP replace those of hClpP; hClpP-N+14, }+4,+2, \text { start with } 14,4 \text {, and } 2 \text {, } \\
\text { respectively, of the preceding amino acids from the mitochondrial targeting sequence of hClpP. } \\
\text { b ClpP folding and heptamer assembly was evaluated by mobility and peak shape upon analytical gel filtration. } \\
{ }^{\mathrm{c}} \text { Peptidase activity was measured by degradation of FAPHMALVPV with ClpP alone or ClpP in Peptidase the presence of excess hClpX or } \\
\text { eClpX (see Section 2). Data are shown for hClpX activation but essentially the same activity was seen with eClpX. } \\
\text { d For protein degradation assays (Section } 2 \text { ) } \alpha \text {-casein was used as the substrate when hClpX was present and } \lambda \mathrm{O} \text { protein was used when eClpX was } \\
\text { present. }\end{array}$} \\
\hline
\end{tabular}


communicated to the active site causing a loss of catalytic activity. The mutants defective in peptidase activity were similarly inactive in ClpX-dependent reactions, including ATP-activated peptide cleavage and ATPdependent protein degradation (Table 2). Protein degradation was blocked with both hClpX- and $\mathrm{eClpX}$-dependent substrates. To determine if the mutations in hClpP-affected interaction with hClpX, complex formation was measured by gel filtration (data not shown) and ultrafiltration assays (Fig. 7A). HClpP$\mathrm{N} \Delta 3$ and hClpP-N $\Delta 15$ bound poorly to $\mathrm{hClpX}$, indicating that the conformation of the N-terminus of ClpP affects its interaction with ClpX. Interestingly, hClpP$\mathrm{N} \Delta 4$, which had very low but measurable activity in the presence of ClpX (Table 2), was able to bind to hClpX (Fig. 7A). These data establish that it is not the $\mathrm{N}$-terminal residues themselves that interact with hClpX but suggest that failure to anchor the $\mathrm{N}$-terminus in the channel perturbs some surface feature of hClpP, interfering with binding to $\mathrm{ClpX}$. The crown loop itself is also not essential for binding to ClpX, because hClpP$\mathrm{N} \Delta 8-15$, in which the crown loop is deleted, folded, and oligomerized properly and could interact, albeit somewhat more weakly, with hClpX (Fig. 7A). HClpP$\mathrm{N} \Delta 8-15$ did not display ClpX-activated peptidase or protease activity (Table 2), indicating that it is lacking an essential element for enzymatic activity.

\subsection{Auto-processing of recombinant hClpP}

The N-terminal proline of the mature hClpP is $34 \AA$ from the active site and $22 \AA$ from a neighboring active site, and thus a major rearrangement would need to occur if maturation of hClpP involves cleavage of the propeptide at this position. To test whether hClpP could undergo auto-processing in the way $E$. coli $\mathrm{ClpP}$ does, we first replaced residues $1-7$ of hClpP with residues 1-21 of eClpP, thereby adding the eClpP propeptide and auto-processing site. When this hybrid, hClpPeN21, was expressed and purified, about $90 \%$ of the protein had an $\mathrm{N}$-terminus of Alal (Fig. 7B), corresponding to the same site of processing observed during maturation of eClpP (Maurizi et al., 1990b). Incubation of the purified protein in vitro resulted in further processing of the remaining propeptide (Fig. 7B), indicating that the $\mathrm{N}$-terminus is inside in the assembled protein. Ala1 aligns with Prol of mature hClpP, implying that, following processing, the N-terminus moves to the channel position observed in the crystal structure of $\mathrm{hClpP}$. The auto-processed product of hClpP-eN21 or

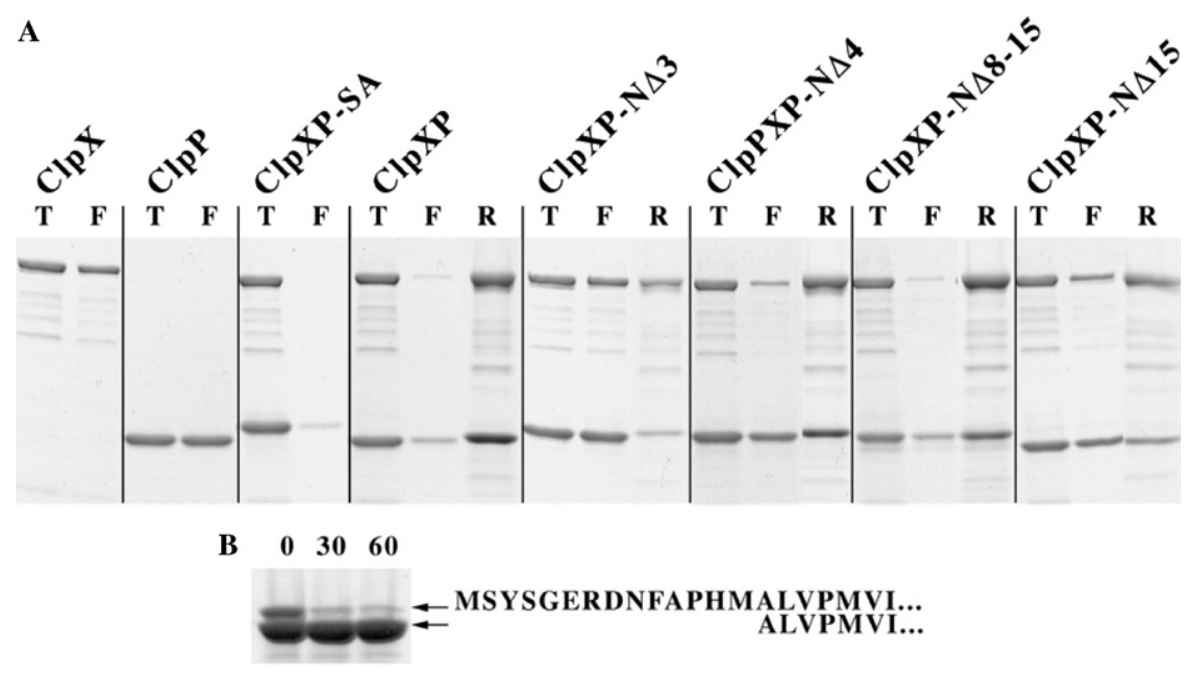

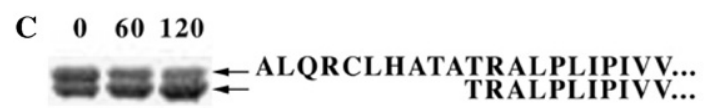

Fig. 7. Interactions between hClpP mutants and hClpX. (A) The ability of hClpP mutants to form stable complexes with hClpX was measured by increased retention upon ultrafiltration through $300 \mathrm{kDa}$ cut-off membranes (see Section 2). HClpX (10 $\mu \mathrm{g})$ and hClpP (10 $\mu \mathrm{g})$, sufficient to allow assembly of 1:1 complexes of hClpX hexamers and hClpP tetradecamers, were passed through Microcon 100 membranes. Equal volumes of original mixture, the filtrate, and the retentate were mixed with SDS sample buffer, run on SDS gels, and stained. The lanes are marked according to the wild type or mutant hClpP used. All mutant hClpP proteins passed through the membranes to a similar degree as wild type hClpP with the exception of hClpP-N $\Delta 8-15$, of which about $50 \%$ was retained. (B) Purified hClpP-eN21, which contains the first 21 amino acids of the eClpP precursor fused to hClpP, is about $90 \%$ processed in E. coli cells. The protein was purified and incubated at $37^{\circ} \mathrm{C}$ for the times shown, after which samples were removed, run on SDS gels, and stained. Parallel samples were blotted to PDVF membranes and sequenced. The hClpP-eN21 sequence starts with Met1. (C) HClpP-N+14, which contains 14 amino acids prior to the mitochondrial processing site, is about $50 \%$ processed in $E$. coli cells. The purified protein was incubated as above and sequenced. The hClpP-N+14 sequence starts with Ala2 (Met1 is processed in vivo). 
a recombinant hClpP-eN7, in which the propeptide was omitted and translation was initiated at $\mathrm{N}$-terminus of the mature protein with the $\mathrm{N}$-terminal seven amino acids of eClpP, both had activities virtually identical to those of wild type hClpP (Table 2).

Preliminary data indicates that processing of mitochondrially targeted hClpP in vivo occurs in two steps, the second of which is dependent on active hClpP itself (Y. Wang, S.G.K., and M.R.M., in preparation). To test whether recombinant $\mathrm{hClpP}$ can process itself, we expressed hClpP-N+14, carrying a 14-amino acid N-terminal extension corresponding to the sequence in the potential hClpP propeptide in vivo. Two products separable by SDS-PAGE were obtained, indicating that partial processing of hClpP occurred in E. coli cells (Fig. $7 \mathrm{C})$. Incubation of the purified partially processed protein at $37^{\circ} \mathrm{C}$ for $2 \mathrm{~h}$, allowed further processing to a maximum of $50-60 \%$; addition of hClpX did not accelerate processing. $\mathrm{N}$-terminal sequencing indicated that the processed form started at $\operatorname{Thr}(-4)$, rather than the expected processing site at Prol (Fig. 7C). The site of auto-processing could be influenced by the length of the pro-region remaining after initial cleavage by the mitochondrial processing protease, which is currently not known. Further analysis of processing and maturation must await determination of the $\mathrm{N}$-terminus of endogenously expressed hClpP.

Both unprocessed hClpP-N+14 and its processed product were active (Table 2 ). We have also noted that hClpP with other $\mathrm{N}$-terminal extensions, such as Histags, are also active. These data are consistent with our structural model showing an inside to outside orientation of the N-terminus, which would place the N-terminal extensions within the chamber. An abundance of data indicates that peptide bond cleavage is not rate limiting for degradation (Burton et al., 2003; Kenniston et al., 2003; Singh et al., 1999; Thompson and Maurizi, 1994; Thompson et al., 1994), and the presence of an extension within the chamber is less likely to interfere with activity than is an extension protruding out on the ClpX-interacting surface.

\subsection{The hClpP active site and surface properties of the chamber}

$\mathrm{HClpP}$ is a serine protease with a catalytic triad consisting of Ser97, His122, and Asp171 disposed similarly to those in subtilisin (Asp32, His64, and Ser221) and chymotrypsin (His57, Asp102, and Ser195). As noted above, the hClpP catalytic triad is located on the inner surface of the chamber at the base of the wedge domain near the tetramerization contacts (Fig. 8A). The active site is coupled to formation of the tetradecamer through the involvement of neighboring residues, all of which are highly conserved. The thin wall of the chamber near the active site is stabilized by a salt bridge between $\operatorname{Arg} 170$
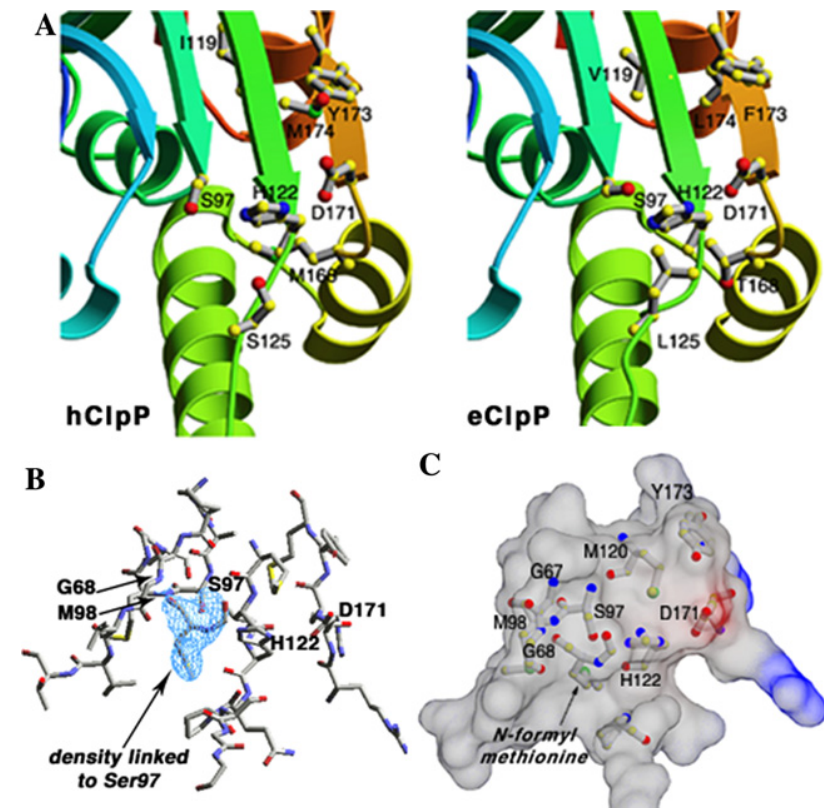

C

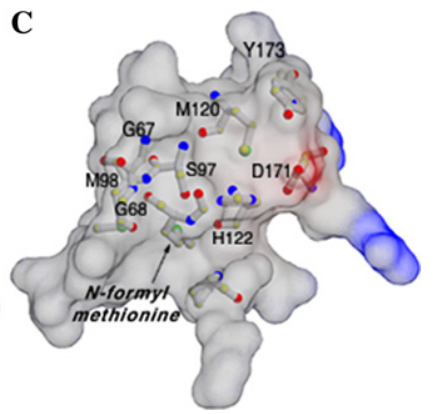

Fig. 8. (A) Comparison of residues in the immediate vicinity of the hClpP (left) and eClpP (right) catalytic triads. Unlike that in eClpP, the hydroxyl of Ser97 in hClpP is positioned to form a proper hydrogen bond with His122. (B) A stick model of the active site of hClpP is shown with a portion of the $3\left|F_{\mathrm{o}}\right|-2\left|F_{\mathrm{c}}\right|$ electron density map contoured at $1 \sigma$ level of a molecule of $N$-formyl methionine covalently bonded to the catalytic residue, Ser97. The residues (Gly68 and Met98) contributing amide nitrogens to stabilize the oxyanion intermediate are also shown. (C) The transparent electrostatic surface potential at the active site from $-15.0 \mathrm{kT}$ (deep red) to $+15.0 \mathrm{kT}$ (deep blue) with the putative $N$-formyl methionine molecule bound in a hydrophobicpocket. The other residues side chains involve to stabilized the oxyanion intermediate have been mapped onto the surface plan.

and Glu169 from subunits facing each other across the 2-fold axis (Fig. 7B). The guanidino groups of the apposing Arg170 side chains approach quite close to Gln123, Gln131, Thr133, Asp134, and Lys146 participate in an interdependent bonding network that influences the positions of Arg170 and Glu169 and, in addition, impinge on the catalytic triad, which is located immediately inside the thin inter-ring contact. The proximity of the catalytic triads to this region suggests that protease activity could be coupled to conformational changes in the wall of the chamber, which may lead to openings between the arms. Whether these side portals would enlarge sufficiently to allow release of peptide products is not yet clear, but this mechanism may provide an alternative to the current problematic model that products exit back through the entry channels.

The presence of the N-terminal residues in the axial channel, together with substitutions of methionine for Thr32 and proline for Gln34 renders the passage to the active site extremely hydrophobic in comparison to the model for eClpP. At the active site, the hydrophobic substrate binding platform found in eClpP is mostly 
conserved with the striking exception of a switch from Leu125 in eClpP to Ser125 in hClpP at the proposed docking site for P2 side chain of the substrate (Fig. 8A). HClpP and eClpP showed similar cleavage specificity, suggesting that the Ser125 site may not be a critical part of the substrate binding groove. The aromatic ring of Tyr123 maintains the hydrophobic position occupied by Phe123 in eClpP, but the hydroxyl introduces a hydrogen bonding interaction to the catalytic Asp171, providing a means by which substrate binding could influence interactions between the catalytic residues. The chamber itself has an unexpected constriction near the center caused by the side chains of Arg129 residues, which project from the arm towards the center of the chamber. In eClpP, this region is unoccupied because Gln129 projects in the opposite direction toward the surface of the tetradecamer. The Arg129 annulus of $\mathrm{hClpP}$ is $\sim 41 \AA$ in diameter and might provide a tethering site for negatively charged regions of substrate polypeptides.

In the crystal reported here and in three others diffracting to $2.2-2.7 \AA$ (data not shown) there was a significant extra density that appeared to be covalently bonded to active site Ser97. The density is observed in both $2\left|F_{\mathrm{o}}\right|-\left|F_{\mathrm{c}}\right|$ and $\left|F_{\mathrm{o}}\right|-\left|F_{\mathrm{c}}\right|$ simulated annealing composite omit electron density maps (Fig. 8B), and was presented in all seven subunits. We note that orientation of the Ser97 hydroxyl in our crystal allows a proper hydrogen bond to be formed with His122-N $\epsilon 2$, which was not the case in the apo form of eClpP, where the serine was not positioned properly for hydrogen bonding (Wang et al., 1997) except in the diisopropylfluoro phosphate-derivatized molecule. The reorientation of the catalytic serine in $\mathrm{hClpP}$ provides further evidence for the covalent nature of the attachment of the extra density. Attempts to identify the group attached to Ser97 have so far been unsuccessful. Mass spectroscopy of full length $\mathrm{hClpP}$ and the trypsin digested protein failed to detect a higher molecular weight species that was consistent with the observed density. While mass spectroscopy did reveal a species with additional mass equal to one methionine, this species is explained by retention of the N-terminal methionine in $\sim 15 \%$ of hClpP expressed in E. coli, which was confirmed by $\mathrm{N}$-terminal sequencing of the purified, protein. The density did not correspond to any known protease inhibitors or to any compounds present in the crystallization solutions. In search of the structure database, we found that an acyl intermediate of $N$-formyl methionine fit reasonably well into the density (Fig. 8C). The pocket near the active site accommodates the structure of the modeled $N$-formyl acyl methionine, and the backbone amides of Met98 (at the C-terminus of $\beta 5$ ) and Gly68 (at the C-terminus of $\beta 4$ ) are involved in hydrogen bonding to the putative acyl carbonyl oxygen. These interactions normally stabilize the tetrahedral oxyanion intermediate during catal- ysis and the same residues were proposed to form the oxyanion hole in eClpP (Wang et al., 1997). As ClpP normally processes its own propeptide at this position, we speculate that $N$-formyl methionine at the active site is the product of incomplete intramolecular processing of the formyl methionine inserted at the N-terminus during biosynthesis. Experiments are underway to confirm the identity of the species and its mechanism of appearance.

\section{Conclusions and biological significance}

The overall similarity in structures of hClpP and eClpP confirms the general mechanism of assembly with chaperone components and compartmentalized degradation characteristic of ATP-dependent proteases (Groll et al., 2000; Lupas et al., 1997) and further suggests that they have similar modes of interaction with ClpX and mechanisms of action. Our model of the $\mathrm{N}$-terminus of hClpP differs from that originally proposed for the $\mathrm{N}$-terminus of eClpP, raising the possibility that these regions may have diverged to contribute slightly different to their respective activities. However, we have presented data indicating that the $\mathrm{N}$-terminus of eClpP may well have a similar configuration as in our hClpP model, and thus it is likely that the N-terminus in fact plays a similar role in both proteins. The high degree of sequence conservation in this region, particularly in residues 2-7 that interact within the channel, is consistent with this conclusion. Our mutational and biochemical data indicate an important function for the N-terminus of hClpP. The position of the $\mathrm{N}$-terminus within the substrate translocation channel suggests that this part of $\mathrm{ClpP}$ might interact with translocating substrates, might contribute to a gating function governing passage of substrates, as is seen with the $\mathrm{N}$-terminal peptides of proteasomal $\alpha$-subunits, or might be needed for the structural integrity of the translocation channel.

The N-terminal peptide of ClpP is mobile, but the presence of defined density confirms that it is fixed within the channel, with the N-terminus directed toward the chamber. The backbone atoms of all seven subunits align reasonably well, although the side chains in two of the seven subunits have variable positions (data not shown). This variability suggests that at least in some configurations the peptide would encounter substrate polypeptides as they pass through the axial channel. Weaker binding of hClpP-N $\Delta 8-15$ to $\mathrm{hClpX}$ suggests that the crown loop either allosterically affects surface docking sites for $\mathrm{ClpX}$ or directly, though weakly, interacts with ClpX. Interactions between the crown loop and $\mathrm{ClpX}$ or ClpA are likely to be communicated to the N-terminal peptides and thereby influence the passage of substrates through the channel. 
The N-terminal peptides of hClpP narrow the axial channel but might not act as a simple passive gate. None of the N-terminal deletions of hClpP led to enhanced peptidase activity, in contrast to the effects of similar mutations in the proteasome, indicating that the N-terminal peptides of ClpP do not simply close the access channel to the active site chamber but either have a direct role in substrate interaction or influence the structure of the channel and affect its substrate interaction properties. Only one mutant (hClpP-P4A) showed any obvious structural change; this mutant formed heptamers but had a tendency to aggregate, possibly because the $\mathrm{N}$-terminal peptide was not properly docked inside the channel. All other mutants formed stable heptamers and many interacted with ClpX. We favor the hypothesis that the defects in the N-terminal mutants reflect altered interactions with substrates. The channel in hClpP is surrounded by acidic and basic residues at the entrance and then is lined with hydrophobic residues further inside. Since many substrates are amphipathic, with alternating stretches of hydrophilic and hydrophobic residues, binding within a conformationally fixed channel might cause blocking of the channel and a delay in translocation. The flexibility of the N-terminal peptide could enable the surface binding properties of the channel to cycle between states with relatively higher or lower affinity for substrates. Such changes, if coordinated with changes in ClpX during cycles of ATP hydrolysis, could prevent substrates remaining in the channel and might facilitate substrate translocation. Interactions between substrates and the channel are expected to be weak and transitory, and further studies will be required to test the possibility of such an active role for the N-terminus.

In the hexamer models of $E$. coli ClpA (Guo et al., 2002) and Helicobacter pylori ClpX (Kim and Kim, 2003), there is a concave antechamber or vestibule on the surface of ClpA facing ClpP. This chamber contains a number of charged residues that are not engaged in intramolecular salt bridges and would thus be available to interact with residues in the crown loop of ClpP. Additional charged residues elsewhere on the ClpP-interacting surface, which are also not engaged in salt bridges, could serve this purpose as well. The asymmetry between heptameric $\mathrm{ClpP}$ and hexameric $\mathrm{ClpX}$ implies that at least one loop of $\mathrm{ClpP}$ will be free or have a different interaction with ClpX. It has been proposed that ClpA (or ClpX) and $\mathrm{ClpP}$ rotate or undergo a reciprocating movement relative to each other during cycles of ATP hydrolysis (Beuron et al., 1998). One prediction is that such movement would result a change in relative position of the crown loops, with a different loop being free following each shift in position. Coordination between engagement of the crown loops and cycles of ATP hydrolysis would, at a minimum, be expected to systematically affect the properties of the translocation channel.
$\mathrm{HClpP}$ without a pro-region is recovered in an active form from E. coli cells. This result is in contrast to the absence of eClpP when constructs lacking the pro-region are expressed. This failure to accumulate depends on $\mathrm{ClpP}$ activity, suggesting that eClpP without its protective propeptide is digested during folding and assembly (S.K. Singh and M.R.M., unpublished). The density found at the active site of hClpP may explain why this protein is recovered. This density if covalently attached to the catalytic serine and may protect $\mathrm{hClpP}$ from autodigestion during assembly. Consequently, hClpP also degrades peptides at only $5 \%$ of the rate as mature eClpP. These data suggest that in mitochondria, hClpP also undergoes self-processing after it is initially processed by the mitochondrial processing protease. Presumably this processing is more efficient in mitochondria and does not leave behind an inhibitory product, which might imply that the pro-region of hClpP can activate its own cleavage. Such a mechanism is consistent with the presence of a number of residues within the substrate binding region that interact with catalytic residues as well. Whether full activity of the ClpP catalytic sites is dependent on the presence of the polypeptide for all substrates remains to be demonstrated, but such dependence would require the $\mathrm{ClpP}$ chamber to take up a minimum amount of substrate before peptide bond cleavage is initiated. The current structure and the mechanistic models it suggests should lead to additional experiments that will shed further light on the workings of this degradative machine.

\section{Acknowledgments}

We thank Zbigniew Dauter, Brookhaven NSLS for assistance with the data collection at $\mathrm{X} 9$ Beam line and Venkatesha Basur, LCB, and NCI for performing the mass spectroscopy.

\section{References}

Barton, G.J., 1993. ALSCRIPT - a tool to format multiple sequence alignments. Protein Eng. 6, 37-40.

Beuron, F., Maurizi, M.R., Belnap, D.M., Kocsis, E., Booy, F.P., Kessel, M., 1998. At sixes and sevens: characterization of the symmetry mismatch of the ClpAP chaperone-assisted protease. J. Struct. Biol. 123, 248-259.

Bochtler, M., Ditzel, L., Groll, M., Huber, R., 1997. Crystal structure of heat shock locus V (HslV) from Escherichia coli. Proc. Natl. Acad. Sci. USA 94, 6070-6074.

Bross, P., Andresen, B.S., Knudsen, I., Kruse, T.A., Gregersen, N., 1995. Human ClpP protease: cDNA sequence, tissue-specific expression and chromosomal assignment of the gene. FEBS Lett. 377, 249-252.

Brunger, A.T., Adams, P.D., Clore, G.M., DeLano, W.L., Gros, P., Grosse-Kunstleve, R.W., Jiang, J.S., Kuszewski, J., Nilges, M., Pannu, N.S.S., et al., 1998. Crystallography \& NMR, system: 
a new software suite for macromolecular structure determination. Acta Crystallogr. D 54, 905-921.

Burton, R.E., Baker, T.A., Sauer, R.T., 2003. Energy-dependent degradation: linkage between ClpX-catalyzed nucleotide hydrolysis and protein-substrate processing. Protein Sci. 12, 893-902.

Burton, R.E., Siddiqui, S.M., Kim, Y.I., Baker, T.A., Sauer, R.T., 2001. Effects of protein stability and structure on substrate processing by the ClpXP unfolding and degradation machine. EMBO J. 20, 3092-3100.

CCP4, 1994. Collaborative Computing Project Number 4. The CCP4 Suite: Programs for protein crystallography. Acta Crystallogr. D 50, 760-763.

Corydon, T.J., Wilsbech, M., Jespersgaard, C., Andresen, B.S., Borglum, A.D., Pedersen, S., Bolund, L., Gregersen, N., Bross, P., 2000. Human and mouse mitochondrial orthologs of bacterial ClpX. Mamm. Genome 11, 899-905.

Gottesman, S., 2003. Proteolysis in bacterial regulatory circuits. Annu. Rev. Cell Dev. Biol. 19, 565-587.

Gottesman, S., Wickner, S., Maurizi, M.R., 1997. Protein quality control: triage by chaperones and proteases. Genes Dev. 11, 815823.

Grimaud, R., Kessel, M., Beuron, F., Steven, A.C., Maurizi, M.R., 1998. Enzymatic and structural similarities between the Escherichia coli ATP-dependent proteases, ClpXP and ClpAP. J. Biol. Chem. 273, 12476-12481.

Groll, M., Bajorek, M., Kohler, A., Moroder, L., Rubin, D.M., Huber, R., Glickman, M.H., Finley, D., 2000. A gated channel into the proteasome core particle. Nat. Struct. Biol. 7, 1062-1067.

Guo, F., Maurizi, M.R., Esser, L., Xia, D., 2002. Crystal structure of ClpA, an Hsp100 chaperone and regulator of ClpAP protease. J. Biol. Chem. 277, 46743-46752.

Halperin, T., Zheng, B., Itzhaki, H., Clarke, A.K., Adam, Z., 2001. Plant mitochondria contain proteolytic and regulatory subunits of the ATP-dependent Clp protease. Plant Mol. Biol. 45, 461-468.

Ishikawa, T., Beuron, F., Kessel, M., Wickner, S., Maurizi, M.R., Steven, A.C., 2001. Translocation pathway of protein substrates in ClpAP protease. Proc. Natl. Acad. Sci. USA 98, 4328-4333.

Jones, T.A., Zou, J.Y., Cowan, S.W., Kjeldgaard, M., 1991. Improved methods for building protein models in electron density maps and the location of errors in these models. Acta Crystallogr. A 47, 110 119.

Kang, S.G., Ortega, J., Singh, S.K., Wang, N., Huang, N.N., Steven, A.C., Maurizi, M.R., 2002. Functional proteolytic complexes of the human mitochondrial ATP-dependent protease, hClpXP. J. Biol. Chem. 277, 21095-21102.

Kenniston, J.A., Baker, T.A., Fernandez, J.M., Sauer, R.T., 2003. Linkage between ATP consumption and mechanical unfolding during the protein processing reactions of an AAA+ degradation machine. Cell 114, 511-520.

Kessel, M., Maurizi, M.R., Kim, B., Kocsis, E., Trus, B.L., Singh, S.K., Steven, A.C., 1995. Homology in structural organization between E. coli ClpAP protease and the eukaryotic a26 S proteasome. J. Mol. Biol. 250, 587-594.

Kim, D.Y., Kim, K.K., 2003. Crystal structure of ClpX molecular chaperone from Helicobacter pylori. J. Biol. Chem. 278, 50664 50670.

Kleywegt, G.J., 1996. Use of non-crystallographic symmetry in protein structure refinement. Acta Crystallogr. D 52, 842-857.
Kleywegt, G.J., Read, R.J., 1997. Not your average density. Structure, $1557-1569$.

Kraulis, P.J., 1991. MOLSCRIPT - a program to produce both detailed and schematic plots of protein structures. J. Appl. Crystallogr. 24, 946-950.

Laskowski, R.A., MacArthur, M.W., Moss, D.S., Thornton, J.M., 1992. PROCHECK: a program to check the stereochemical quality of protein structures. J. Appl. Crystallogr. 26, 283-291.

Lowe, J., Stock, D., Jap, B., Zwickl, P., Baumeister, W., Huber, R., 1995. Crystal structure of the a20S proteasome from the archaeon T. acidophilum at 3.4 A resolution. Science 268, 533-539.

Lupas, A., Flanagan, J.M., Tamura, T., Baumeister, W., 1997. Selfcompartmentalizing proteases. Trends Biochem. Sci. 22, 399-404.

Maurizi, M.R., Clark, W.P., Katayama, Y., Rudikoff, S., Pumphrey, J., Bowers, B., Gottesman, S., 1990a. Sequence and structure of Clp P, the proteolytic component of the ATP-dependent Clp protease of Escherichia coli. J. Biol. Chem. 265, 12536-12545.

Maurizi, M.R., Clark, W.P., Kim, S.H., Gottesman, S., 1990b. Clp P represents a unique family of serine proteases. J. Biol. Chem. 265, 12546-12552.

Merritt, E.A., Bacon, D.J., 1997. Raster3D: photorealistic molecular graphics. Methods Enzymol. 277, 505-525.

Navaza, J., Saludjian, P., 1997. aMoRe: an automated molecular replacement program package. Methods Enzymol. 276, 581-594.

Neuwald, A.F., Aravind, L., Spouge, J.L., Koonin, E.V., 1999. AAA+: a class of chaperone-like ATPases associated with the assembly, operation, and disassembly of protein complexes. Genome Res. 9, 27-43.

Nicholls, A., Sharp, K.A., Hoing, B., 1991. Protein folding association: insights from the interfacial and thermodynamic properties of hydrocarbons. Protein Eng. 11, 281-296.

Ortega, J., Singh, S.K., Ishikawa, T., Maurizi, M.R., Steven, A.C., 2000. Visualization of substrate binding and translocation by the ATP-dependent protease, ClpXP. Mol. Cell 6, 1515-1521.

Otwinowski, Z., Minor, W., 1997. Processing of X-ray diffraction data collecting in oscillation mode. Methods Enzymol. 276, 307-326.

Schelin, J., Lindmark, F., Clarke, A.K., 2002. The clpP multigene family for the ATP-dependent Clp protease in the cyanobacterium Synechococcus. Microbiology 148, 2255-2265.

Schirmer, E.C., Glover, J.R., Singer, M.A., Lindquist, S., 1996. HSP100/Clp proteins: a common mechanism explains diverse functions. Trends Biochem. Sci. 21, 289-296.

Singh, S.K., Guo, F., Maurizi, M.R., 1999. ClpA and ClpP remain associated during multiple rounds of ATP-dependent protein degradation by ClpAP protease. Biochemistry 38, 14906-14915.

Sousa, M.C., Trame, C.B., Tsuruta, H., Wilbanks, S.M., Reddy, V.S., McKay, D., 2000. Crystal and solution structures of an HsIUV protease-chaperone complex. Cell 103, 633-643.

Thompson, M.W., Maurizi, M.R., 1994. Activity and specificity of Escherichia coli ClpAP protease in cleaving model peptide substrates. J. Biol. Chem. 269, 18201-18208.

Thompson, M.W., Singh, S.K., Maurizi, M.R., 1994. Processive degradation of proteins by the ATP-dependent Clp protease from Escherichia coli. Requirement for the multiple array of active sites in ClpP but not ATP hydrolysis. J. Biol. Chem. 269, 18209-18215.

Wang, J., Hartling, J.A., Flanagan, J.M., 1997. The structure of ClpP at 2.3 A resolution suggests a model for ATP-dependent proteolysis. Cell 91, 447-456. 